\title{
The 5-Lipoxygenase as a Common Pathway for Pathological Brain and Vascular Aging
}

\author{
Jin Chu and Domenico Praticò \\ Department of Pharmacology School of Medicine, Temple University, Philadelphia, PA 19140, USA \\ Correspondence should be addressed to Domenico Praticò, praticod@temple.edu
}

Received 19 May 2009; Accepted 29 June 2009

Recommended by Hari Manev

\begin{abstract}
Epidemiological studies indicate age as a strong risk factor for developing cardiovascular and neurodegenerative diseases. During the aging process, changes in the expression of particular genes can influence the susceptibility to these diseases. 5-Lipoxygenase (5-LO) by oxidizing fatty acids forms leukotrienes, potent mediators of oxidative and inflammatory reactions, two key pathogenic events in both clinical settings. This enzyme is widely distributed in the cardiovascular as well as in the central nervous system, where its expression levels increase with age, suggesting that it may be involved in their diseases of aging. The central theme of this article is that during aging, 5-LO acts as biologic link between different stressors and the development of cardiovascular and neurodegenerative diseases. We hypothesize that the age-dependent upregulation of 5-LO represents a "priming" factor in the vasculature as well as in the brain, where a subsequent exposure to triggering stimuli (i.e., infections) leads to an abnormal chronic inflammatory reaction, and ultimately results in increased organ vulnerability and functional deficits.
\end{abstract}

Copyright ( $\odot 2009$ J. Chu and D. Praticò. This is an open access article distributed under the Creative Commons Attribution License, which permits unrestricted use, distribution, and reproduction in any medium, provided the original work is properly cited.

\section{Introduction}

Consistent demographic data show that due to the improvements in public health and advances in medical therapy, the number of older people (over 65 years) is fast increasing worldwide, and it is expected to triple by 2040 .

Since advancing age is the strongest risk factor for developing chronic diseases, the burden from them is expected to increase several-fold over the next 15-20 years. This fact has created a sense of emergency toward this segment of the population in view of the potential catastrophic socioeconomic consequences. Interestingly, age is a nonmodifiable risk factor for atherosclerosis and chronic neurodegenerative diseases such as Alzheimer's disease (AD) $[1,2]$. The aging process is the most common feature of the postreproductive phase of life. It manifests in all multicellular organisms and is characterized by a progressive reduction in the efficacy of a number of physiological processes. This decline translates to a reduced capacity to maintain homeostatic control of important functions and finally results in increased organ vulnerability.
In experimental models, for example, aged animals have an exacerbation to experimental vascular injury and develop atherosclerosis even on a chow diet [3]. On the other hand, they also exhibit an impaired ability to sustain long-term potentiation, a form of synaptic plasticity that has been proposed as biological substrate for learning and/or memory [4], and have impaired spatial learning in the Morris water maze [5].

\section{The 5-LO Pathway in the Vasculature and Central Nervous System}

5-Lipoxygenase (5-LO) is a member of a large family of enzymes, called lipoxygenases, which oxidizes free and esterified polyunsaturated fatty acids. 5-LO first introduces active molecular oxygen to carbon 5 of arachidonic acid resulting in the formation of 5-Hydroxy-peroxy-eicosatetraenoic acid (5HPETE). This unstable derivative is either reduced to 5-Hydroxy-eicosatetraenoic acid (5HETE), or converted to leukotriene (LT) A4. However, LTA4 can serve either as an intracellular intermediate in the synthesis of LTB4 and 


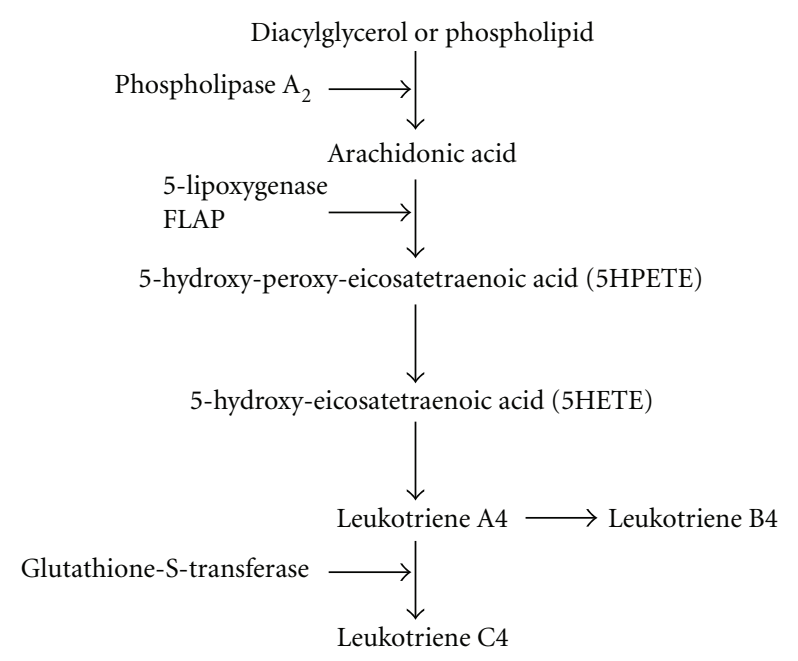

FIGURE 1: Schematic representation of the 5-Lipoxygenase enzyme metabolic pathway. Arachidonic acid is released from diacyglycerol or membrane phospholipids via the action of Phospholipase $A_{2}$. Once free, arachidonic acid is oxidized by 5-lipoxygenase (5-LO), which has been activated by the Five-Lipoxygenase-ActivatingProtein (FLAP), at carbon 5 to form the unstable 5-hydroxy-peroxyeicosatetraenoic acid (5HPETE), which is promptly metabolized into the more stable 5-hydroxy-eicosatetraneoic acid (5HETE). The 5HETE can then be converted in leukotriene A4 (LTA4), which can serve either as an intracellular intermediate in the synthesis of LTB4 and LTC4, or may be released extracellularly and subsequently be taken up by adjacent cells devoid of 5-LO activity but expressing LTA4-hydrolase and/or LTC4 synthase.

LTC4, or may be released extracellularly and subsequently be taken up by adjacent cells devoid of 5-LO activity but expressing LTA4-hydrolase and/or LTC4 synthase. LTs and the cysteinyl derivatives of LTs all have strong pro-oxidant and proinflammatory activities [6] (see Figure 1).

5-LO is widely expressed in the cardiovascular system, that is, aorta, coronary, and carotid arteries, as well as in macrophages and neutrophils. Interestingly, its expression levels are increased in aortas of old animals when compared with young ones [7]. This enzymatic pathway is also widely expressed in the central nervous system (CNS), where it localizes mainly in neuronal cells of the hippocampus and cortex, and, similar to the vasculature, its levels increase significantly with aging $[8,9]$.

The expression of 5-LO is susceptible to hormonal regulation, since higher levels are observed in conditions of melatonin deficiency and/or hyperglucocorticoidemia [10, 11], both of which are common in elderly subjects [12]. Although in general upregulation of 5-LO might serve a physiological purpose, during the aging process, it may also increase the vulnerability of the cardiovascular system and CNS to different insults/stressors [13]. Given that older subjects are at greater risk of health complications and mortality stemming from altered inflammatory and immune functions, and aging, via the upregulation of 5-LO, can be an important risk factor, the effects of stressors on this enzymatic pathway are of particular importance.

\section{5-LO, Aging and Cardiovascular Diseases}

Recent studies have implicated 5-LO in the pathogenesis of atherosclerosis [14], and have also identified specific 5-LO genotypes in subpopulations with increased risk of atherosclerosis $[15,16]$.

Age is an established risk factor for atherosclerosis. Among primates and rodents, older animals develop more extensive atherosclerosis than younger animals $[17,18]$. Ageaccelerated vascular injury is commonly considered to result from increased oxidative stress, leading to inflammation and endothelial dysfunction [19]. Tissues from aged animals demonstrate increase generation of reactive oxygen species (ROSs) that lead to damage to vascular cells with ageassociated remodeling changes, and oxidation of lipids, that is, leukotrienes, with potent proinflammatory and proatherogenic actions $[20,21]$. Interestingly, in experimental models of atherogenesis, the disease process can be exacerbated by inflammatory stress such as lipopolysaccharide (LPS) exposure [22-24]. LPS binds to the Toll-like receptor 4 (TLR4) on the surface of a variety of cell types stimulating, among other things, the generation of inflammatory leukotrienes derived from the 5-LO pathway [25]. In this setting, pharmacologic blockade of this enzyme or its genetic deficiency affords a significant protective effect against organ injury and dysfunction [26]. These facts, together with the upregulation of 5-LO in the aging vasculature support the hypothesis that this enzymatic pathway plays a functional role in the development of aging-related cardiovascular diseases.

\section{5-LO, Aging and Neurodegenerative Diseases}

In the CNS, aging, in general, is associated with an increased incidence of chronic neurodegenerative processes, and among them, $\mathrm{AD}$ is the most frequent $[27,28]$. From a biochemical point of view, brain aging is often associated with microglia activation and a diffuse and chronic brain inflammation involving also other cell types, that is, neurons [29]. Interestingly, aged animals show greater increase in central inflammatory cytokines compared with young adults following both peripheral and central LPS administration $[30,31]$. This response is accompanied by a greater deficit in spatial working memory than is seen in young adult mice [32, 33], and suggests a possible modulatory role for 5-LO. Thus, as stress appears to sensitize the CNS to subsequent insults, so may aging with the upregulation of 5-LO sensitizes cells of the immune system to stress itself. Although there is evidence that stress can influence immune responses and memory performance in elderly, however, the direct effect of 5-LO on these phenomena is still largely unknown. In the aging brain, the prolonged stress-dependent inflammation status could then serve as precursor for augmented neuronal vulnerability which often culminates in cell death and loss of function. Beside the inflammatory hypothesis, recent work has also highlighted a novel concept that 5-LO can directly modulate neurotransmitter receptors as well as amyloid beta peptide metabolism, both of which are well-established mechanisms involved in brain aging $[34,35]$. 


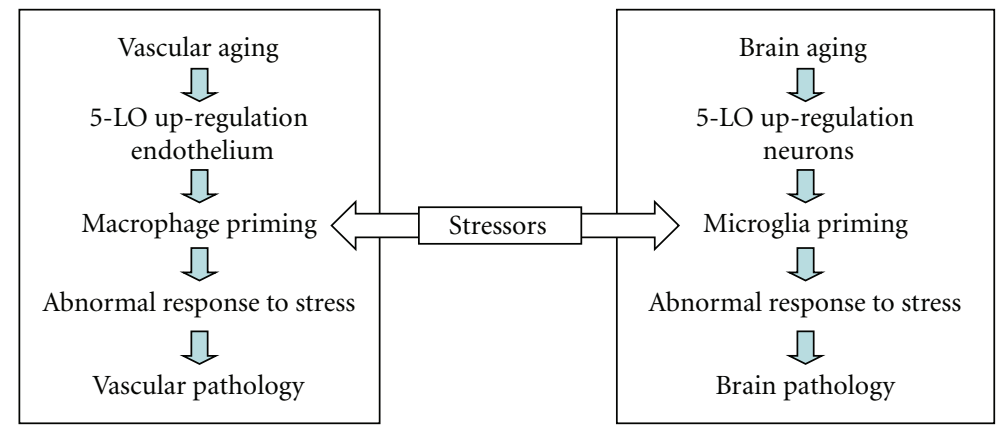

FIGURE 2: Hypothetical model whereby 5-Lipoxygenase influences brain and vascular pathological aging. During aging, peripheral and central stressors targeting the vasculature and/or the central nervous system find these organs primed to a chronic inflammatory status secondary to the upregulation of 5-LO in endothelial cells and macrophages, neurons, and microglia, respectively. This fact facilitates an abnormal and long-lasting inflammatory response, which ultimately results in increased organ vulnerability, functional impairments, and development of pathology.

\section{Peripheral Stressors: Effect on Cardio- vascular and Neurodegenerative Diseases}

Stress is a risk factor for pathological aging because elderly individuals prone to psychological distress are more likely to develop cardiovascular and/or neurodegenerative disorders than age-matched controls, nonstressed individuals. Recent studies suggest that activation of peripheral immune system elicits a discordant inflammatory response in aged but, otherwise, healthy subjects compared with younger cohorts, and the reactive state of immune cells in the aged individuals has been suggested as the basis for this abnormal inflammatory response. We hypothesize that the upregulation of 5-LO in the aging brain and vasculature by releasing high amount of leukotrienes functions as priming factor for these organs facilitating an abnormal inflammatory response to stressors, which ultimately results in increased organ vulnerability and functional impairments (Figure 2).

Importantly, while these responses are transient and reversible in young, by contrast they are generally exacerbated and long-lasting in aged subjects.

In what follows, we briefly discuss two models of stress which have been widely used in the aging field. Both of them mimick in vivo biologically relevant situations: LPS, as bacterial infection (very frequent in elderly); high levels of glucocorticosteroids (as it is typically observed in aging) $[36,37]$.

\section{LPS}

Administration of LPS has been widely used as a model to trigger both vascular and neuroinflammatory responses [3841]. These inflammatory responses, in part mediated by 5 LO activation, could act in concert with aging to accelerate vascular and neuronal vulnerability and subsequent cell loss. It is conceivable that the 5-LO upregulation in endothelial cells and neurons, typical of the aging process, can function as priming event toward macrophages/microglia in these systems and sensitizes them to an increased susceptibility and abnormal biological response to stressors (Figure 2).

\section{Glucocorticoids}

Recent data suggest that glucocorticoid-sensitive mecha$\operatorname{nism}(\mathrm{s})$ are operative in increasing neuronal vulnerability of the aging brain. Thus, high glucocorticoid levels appear to be a constant feature of senile dementia [42]. Aged humans with prolonged elevated levels of cortisol exhibit reduced hippocampal volume and deficits in hippocampus-dependent memory tasks compared with normal cortisol controls. Further, glucocorticoids can negatively affect neuronal survival [43] in vitro, and impairs cognition in vivo [44]. Similarly, a dysregulated cortisol secretion, may be secondary to abnormalities in the hypothalamic-pituitary-adrenal axis, has also been involved in the failure to contain inflammatory reactions within the vasculature $[45,46]$. In both scenarios, the age-dependent 5-LO upregulation could further sensitize these organs to glucocorticoid-mediated detrimental effects (Figure 2).

In both cases, the hypothesis could be easily tested considering the availability of different selective inhibitors of this enzymatic pathway, together with mice which are genetically deficient for this enzyme. Thus, treating aged animals with LPS or corticosteroids in the presence of the inhibitors, or aged 5-LO knock-out and wild-type mice with the same stressors could provide us with important information supporting the functional role of this metabolic pathway.

\section{Conclusions}

Because of the projected aging of the human population, the burden from diseases of aging is expected to increase dramatically over the next 20-25 years. The identification of a putative common molecular mechanism influencing these diseases and amenable of a therapeutic modulation would result not only in an improvement of the quality of life for this segment of the population but also in a significantly reduced socio-economic impact of these diseases. The fact that the 5-LO is significantly increased with aging, which associates with the development of cardiovascular as well as 
neurodegenerative diseases, makes this enzymatic pathway an excellent candidate that fulfills these criteria.

Several molecular mechanisms have been invoked for the 5-LO-mediated age-dependent increased cardiovascular risk, and most of them involve modulation of the inflammatory vascular response to stressors. By contrast, much less is known about the molecular mechanisms operating in the 5LO-mediated pathologic brain aging. Beside the role of 5-LO in regulating neuroinflammation, more recent works have pointed out some novel mechanisms and pathways whereby this enzyme may be involved in pathological brain aging, that is, neurotransmitter receptors and amyloid beta peptide metabolism.

Future studies are warranted to provide a more conclusive evidence for this association, and new or revisited common molecular mechanisms responsible for it.

\section{References}

[1] S. S. Najjar, A. Scuteri, and E. G. Lakatta, "Arterial aging: is it an immutable cardiovascular risk factor?" Hypertension, vol. 46, no. 3, pp. 454-462, 2005.

[2] M. Goedert and M. G. Spillantini, "A century of Alzheimer's disease," Science, vol. 314, no. 5800, pp. 777-781, 2006.

[3] X. Zhang, R. Qi, X. Xian, et al., "Spontaneous atherosclerosis in aged lipoprotein lipase-deficient mice with severe hypertriglyceridemia on a normal chow diet," Circulation Research, vol. 102, no. 2, pp. 250-256, 2008.

[4] M. A. Lynch, "Age-related impairment in long-term potentiation in hippocampus: a role for the cytokine, interleukin-1 $\beta$ ?" Progress in Neurobiology, vol. 56, no. 5, pp. 571-589, 1998.

[5] K. Sugaya, M. Chouinard, R. Greene, et al., "Molecular indices of neuronal and glial plasticity in the hippocampal formation in a rodent model of age-induced spatial learning impairment," Journal of Neuroscience, vol. 16, no. 10, pp. 34273443, 1996.

[6] A. R. Brash, "Lipoxygenases: occurrence, functions, catalysis, and acquisition of substrate," Journal of Biological Chemistry, vol. 274, no. 34, pp. 23679-23682, 1999.

[7] Y. Zou, D. H. Kim, K. J. Jung, et al., "Lysophosphatidylcholine enhances oxidative stress via the 5-lipoxygenase pathway in rat aorta during aging," Rejuvenation Research, vol. 12, no. 1, pp. 15-24, 2009.

[8] C.-H. Lammers, P. Schweitzer, P. Facchinetti, et al., "Arachidonate 5-lipoxygenase and its activating protein: prominent hippocampal expression and role in somatostatin signaling," Journal of Neurochemistry, vol. 66, no. 1, pp. 147-152, 1996.

[9] C. M. Chinnici, Y. Yao, and D. Praticò, "The 5-lipoxygenase enzymatic pathway in the mouse brain: young versus old," Neurobiology of Aging, vol. 28, no. 9, pp. 1457-1462, 2007.

[10] T. Uz, P. Longone, and H. Manev, "Increased hippocampal 5lipoxygenase mRNA content in melatonin-deficient, pinealectomized rats," Journal of Neurochemistry, vol. 69, no. 5, pp. 2220-2223, 1997.

[11] T. Uz, Y. Dwivedi, A. Qeli, M. Peters-Golden, G. Pandey, and H. Manev, "Glucocorticoid receptors are required for upregulation of neuronal 5-lipoxygenase (5-LOX) expression by dexamethasone," The FASEB Journal, vol. 15, no. 10, pp. 17921794, 2001.
[12] F. Magri, M. Locatelli, G. Balza, et al., "Changes in endocrine circadian rhythms as markers of physiological and pathological brain aging," Chronobiology International, vol. 14, no. 4, pp. 385-396, 1997.

[13] M. G. Frank, M. V. Baratta, D. B. Sprunger, L. R. Watkins, and S. F. Maier, "Microglia serve as a neuroimmune substrate for stress-induced potentiation of CNS pro-inflammatory cytokine responses," Brain, Behavior, and Immunity, vol. 21, no. 1, pp. 47-59, 2007.

[14] M. Mehrabian, H. Allayee, J. Wong, et al., "Identification of 5-lipoxygenase as a major gene contributing to atherosclerosis susceptibility in mice," Circulation Research, vol. 91, no. 2, pp. 120-126, 2002.

[15] J. H. Dwyer, H. Allayee, K. M. Dwyer, et al., "Arachidonate 5-lipoxygenase promoter genotype, dietary arachidonic acid, and atherosclerosis," New England Journal of Medicine, vol. 350, no. 1, pp. 29-37, 2004.

[16] A. Helgadottir, A. Manolescu, G. Thorleifsson, et al., "The gene encoding 5-lipoxygenase activating protein confers risk of myocardial infarction and stroke," Nature Genetics, vol. 36, no. 3, pp. 233-239, 2004.

[17] K. W. Weingand, T. B. Clarkson, M. R. Adams, and A. D. Bostrom, "Effects of age and/or puberty on coronary artery atherosclerosis in cynomolgus monkeys," Atherosclerosis, vol. 62, no. 2, pp. 137-144, 1986.

[18] L. G. Spagnoli, A. Orlandi, A. Mauriello, et al., "Aging and atherosclerosis in the rabbit. 1. Distribution, prevalence and mosphology of atherosclerotic lesions," Atherosclerosis, vol. 89, no. 1, pp. 11-24, 1991.

[19] J. E. Mcewen, P. Zimniak, J. L. Mehta, and R. J. Shookler Reis, "Molecular pathology of aging and its implications for senescent coronary atherosclerosis," Current Opinion in Cardiology, vol. 20, no. 5, pp. 399-406, 2005.

[20] N. R. Madamanchi and M. S. Runge, "Mitochondrial dysfunction in atherosclerosis," Circulation Research, vol. 100, no. 4, pp. 460-473, 2007.

[21] A. Csiszar, N. Labinskyy, X. Zhao, et al., "Vascular superoxide and hydrogen peroxide production and oxidative stress resistance in two closely related rodent species with disparate longevity," Aging Cell, vol. 6, pp. 783-797, 2007.

[22] E. Lalla, I. B. Lamster, M. A. Hofman, et al., "Oral infection with a periodontal pathogen accelerates early atherosclerosis in apolipoprotein E-null mice," Arteriosclerosis, Thrombosis, and Vascular Biology, vol. 23, pp. 1405-1411, 2003.

[23] L. Li, E. L. Batista Jr., R. A. Levine, et al., "Porphyromonas gingivalis infection accelerates the progression of atherosclerosis in heterozygous apolipoprotein E-deficient murine model," Circulation, vol. 105, pp. 861-867, 2002.

[24] D. Recalde, M. A. Ostos, E. Badell, et al., "Human apolipoprotein A-IV reduces secretion of proinflammatory cytokines and atherosclerotic effects of a chronic infection mimicked by lipopolysaccharides," Arteriosclerosis, Thrombosis, and Vascular Biology, vol. 24, pp. 756-761, 2004.

[25] K. J. Serio, K. V. Reddy, and T. D. Bigby, "Lipopolysaccharide induces 5-lipoxygenase-activating protein gene expression in THP-1 cells via a NF- $\kappa$ B and C/EBP-mediated mechanism," American Journal of Physiology, vol. 288, no. 5, pp. C1125C1133, 2005.

[26] M. Collin, A. Rossi, S. Cuzzocrea, et al., "Reduction of the multiple organ injury and dysfunction caused by endotoxinemia in 5-lipoxygenase knockout mice and by the 5-lipoxygenase inhibitor zileuton," Journal of Leukocyte Biology, vol. 76, pp. 961-970, 2004. 
[27] P. L. McGeer and E. G. McGeer, "Inflammation and the degenerative diseases of aging," Annals of the New York Academy of Sciences, vol. 1035, pp. 104-116, 2004.

[28] D. Praticò, "Alzheimer's disease and oxygen radicals: new insights," Biochemical Pharmacology, vol. 63, no. 4, pp. 563$567,2002$.

[29] T. B. L. Kirkwood, M. Feder, C. E. Finch, et al., "What accounts for the wide variation in life span of genetically identical organisms reared in a constant environment?" Mechanisms of Ageing and Development, vol. 126, no. 3, pp. 439-443, 2005.

[30] J. Chen, J. B. Buchanan, N. L. Sparkman, J. P. Godbout, G. G. Freund, and R. W. Johnson, "Neuroinflammation and disruption in working memory in aged mice after acute stimulation of the peripheral innate immune system," Brain, Behavior, and Immunity, vol. 22, no. 3, pp. 301-311, 2008.

[31] J. P. Godbout, J. Chen, J. Abraham, A. F. Richwine, B. M. Berg, K. W. Kelley, and R. W. Johnson, "Exaggerated neuroinflammation and sickness behavior in aged mice following activation of the peripheral innate immune system," FASEB Journal, vol. 19, no. 10, pp. 1329-1331, 2005.

[32] G. Riccioni, C. Di Ilio, P. Conti, T. C. Theoharides, and N. D'Orazio, "Advances in therapy with antileukotriene drugs," Annals of Clinical and Laboratory Science, vol. 34, no. 4, pp. 379-387, 2004.

[33] M. I. Combrinck, V. H. Perry, and C. Cunningham, "Peripheral infection evokes exaggerated sickness behaviour in preclinical murine prion disease," Neuroscience, vol. 112, no. 1, pp. 7-11, 2002.

[34] M. Imbesi, I. Zavoreo, T. Uz, et al., "5-lipoxygenase inhibitor MK-886 increases GluR1 phosphorylation in neuronal cultures in vitro and in the mouse cortex in vivo," Brain Research, vol. 1147, no. 1, pp. 148-153, 2007.

[35] O. Firuzi, J. Zhuo, C. M. Chinnici, T. Wisniewski, and D. Praticò, "5-lipoxygenase gene disruption reduces amyloid- $\beta$ pathology in a mouse model of Alzheimer's disease," FASEB Journal, vol. 22, no. 4, pp. 1169-1178, 2008.

[36] J. G. Csernansky, H. Dong, A. M. Fagan, et al., "Plasma cortisol and progression of dementia in subjects with Alzheimer's disease-type dementia," The American Journal of Psychiatry, vol. 163, pp. 2164-2169, 2006.

[37] R. S. Wilson, S. E. Arnold, J. A. Schneider, J. F. Kelly, Y. Tang, and D. A. Bennett, "Chronic psychological distress and risk of Alzheimer's disease in old age," Neuroepidemiology, vol. 27, no. 3, pp. 143-153, 2006.

[38] C. Cunningham, D. C. Wilcockson, S. Campion, K. Lunnon, and V. H. Perry, "Central and systemic endotoxin challenges exacerbate the local inflammatory response and increase neuronal death during chronic neurodegeneration," Journal of Neuroscience, vol. 25, no. 40, pp. 9275-9284, 2005.

[39] M. D. Nguyen, T. D’Aigle, G. Gowing, J.-P. Julien, and S. Rivest, "Exacerbation of motor neuron disease by chronic stimulation of innate immunity in a mouse model of amyotrophic lateral sclerosis," Journal of Neuroscience, vol. 24, no. 6, pp. 1340-1349, 2004.

[40] M. A. Ostos, D. Recalde, M. M. Zakin, and D. Scott-Algara, "Implication of natural killer T cells in atherosclerosis development during LPS-induced chronic inflammation," FEBS Letters, vol. 519, pp. 23-29, 2002.

[41] S. K. Heo, H. J. Yun, E. K. Noh, W. H. Park, and S. D. Park, "LPS induces inflammatory responses in human aortic vascular smooth muscle cells via toll-like receptor 4 expression and nitric oxide production," Immunology Letters, vol. 120, pp. 57-64, 2008.
[42] G. R. Swanwick, M. Kirby, I. Bruce, et al., "Hypothalalmicpituitary-adrenal axis dysfunction in Alzheimer's disease: lack of association between longitudinal and cross-sectional findings," The American Journal of Psychiatry, vol. 155, pp. 286-289, 1998.

[43] B. Stein-Behrens, M. P. Mattson, I. Chang, M. Yeh, and R. Sapolsky, "Stress exacerbates neuronal loss and cytoskeletal pathology in the hippocampus," The Journal of Neuroscience, vol. 14, pp. 5373-5380, 1994.

[44] P. S. Aisen, K. L. Davis, J. D. Berg, et al., "A randomized controlled trial of prednisone in Alzheimer's disease," Neurology, vol. 54, pp. 588-593, 2000.

[45] J. Nijm and L. Jonasson, "Inflammation and cortisol response in coronary artery disease," Annals of Internal Medicine, vol. 41, pp. 224-233, 2009.

[46] O. Radmark and B. Samulesson, "5-lipoxygenase: regulation and possible involvement in atherosclerosis," Prostaglandins \& Other Lipid Mediators, vol. 83, pp. 162-174, 2007. 


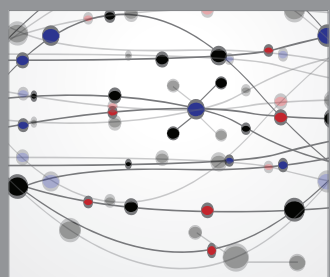

The Scientific World Journal
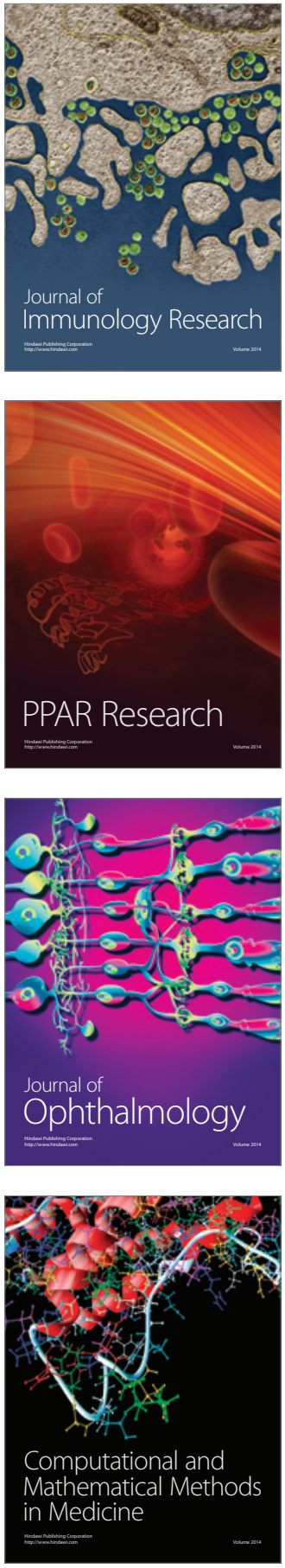

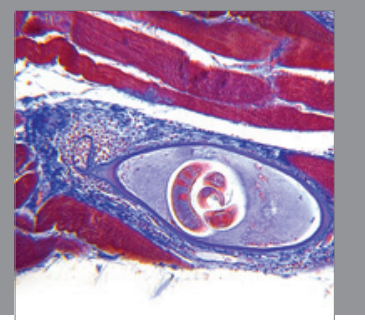

Gastroenterology

Research and Practice
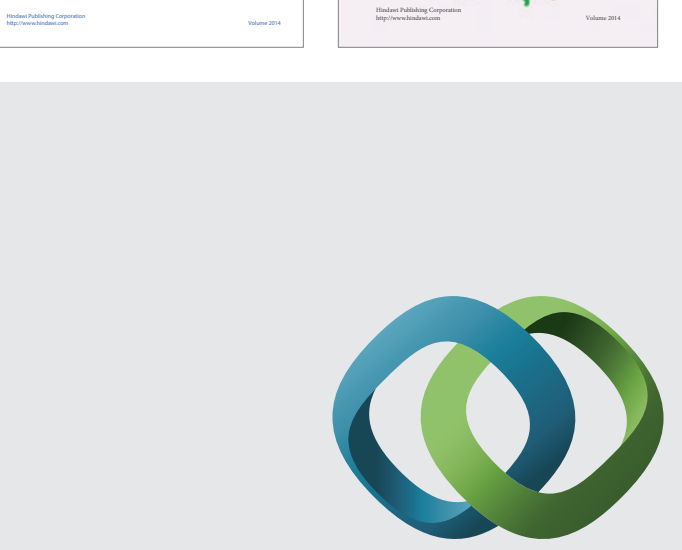

\section{Hindawi}

Submit your manuscripts at

http://www.hindawi.com
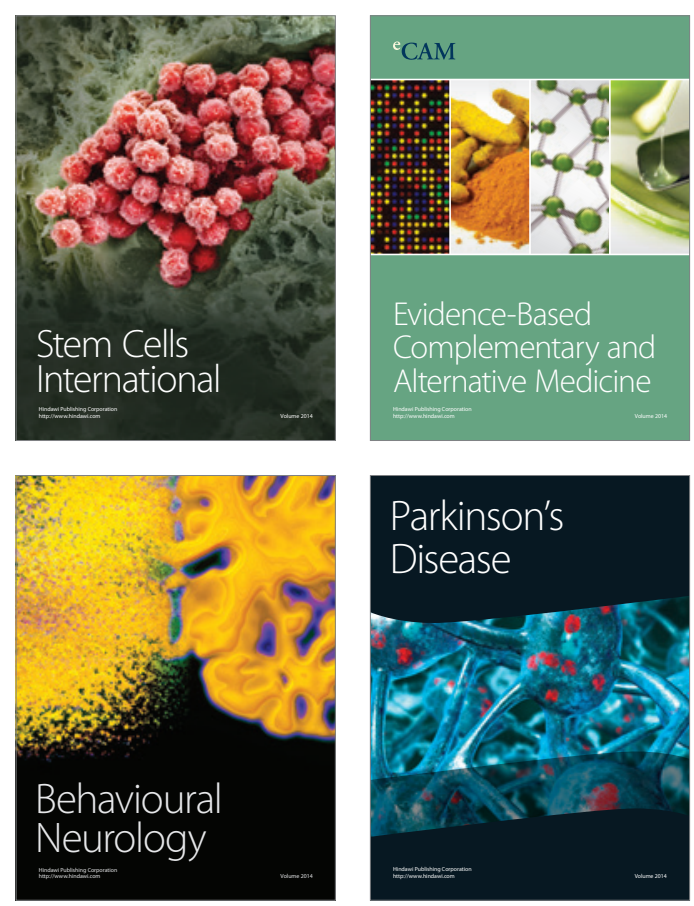

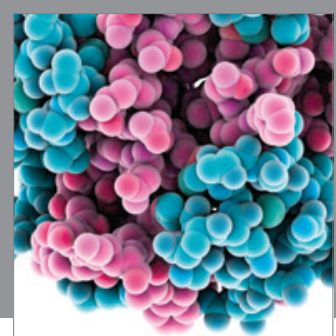

Journal of
Diabetes Research

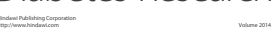

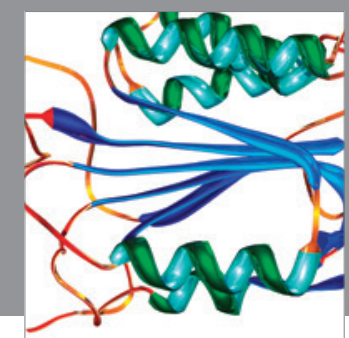

Disease Markers
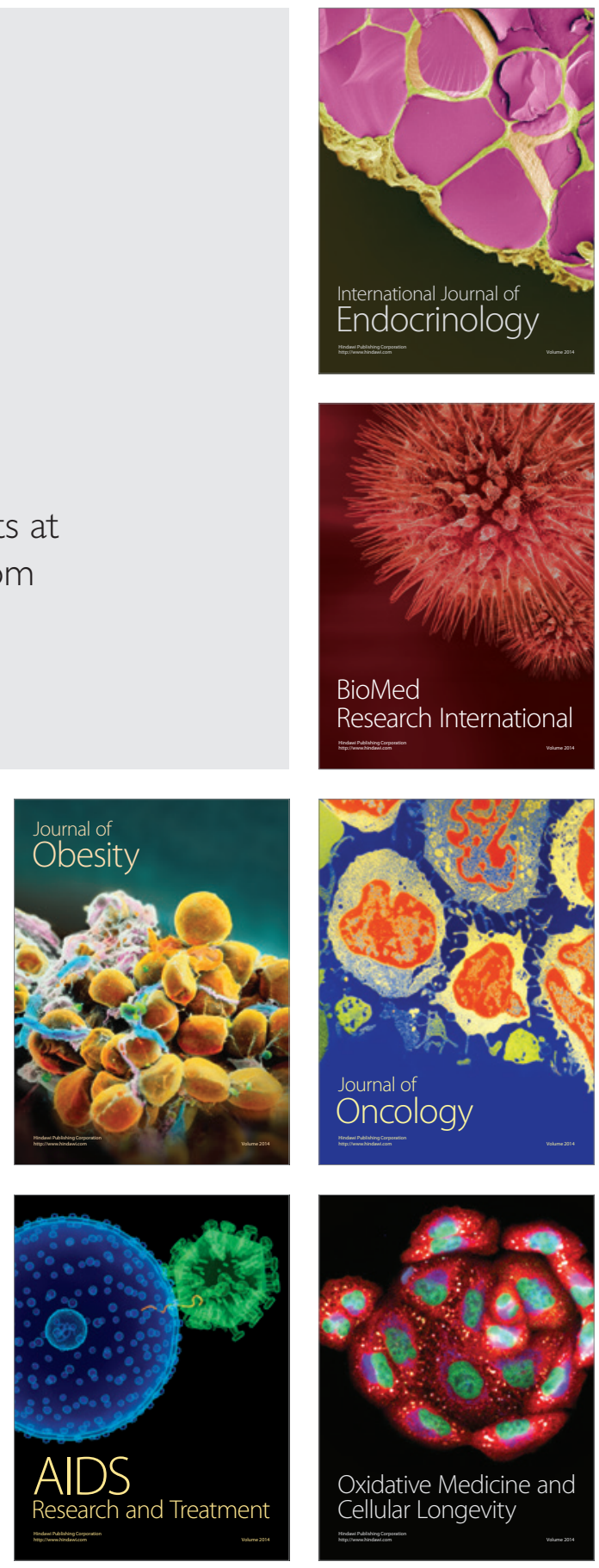